\title{
Development of Real-time Boron Nitride Erosion Monitoring System for Hall Thrusters by Cavity Ring-Down Spectroscopy
}

\author{
By Naoji YAMAMOTO ${ }^{1)}$, Azer P.YALIN ${ }^{2)}$, Lei TAO ${ }^{2)}$, Timothy B. SMITH ${ }^{3)}$, Alec D. GALLIMORE ${ }^{3)}$, and \\ Yoshihiro ARAKAWA ${ }^{4)}$ \\ 1) Department of Advanced Energy Engineering Science, Kyushu University, Kasuga, Japan \\ ${ }^{2)}$ Department of Mechanical Engineering, Colorado State University, Fort Collins, USA \\ ${ }^{3)}$ Department of A Aerospace Engineering, University of Michigan, Ann Arbor, USA \\ 4) Department of Aeronautics and Astronautics, The University of Tokyo, Tokyo, Japan
}

(Received April 25th, 2008)

\begin{abstract}
Sputter monitoring system using continuous-wave cavity ring-down spectroscopy (cw-CRDS) was built for both lifetime assessment and contamination effects in Hall thrusters. First, we have performed proof of principle measurements of sputtered boron atoms from BN targets by argon ions using pulsed CRDS. The measurement strategy is based upon detection of boron atoms via an absorption line from ground state at a wavelength of $249.773 \mathrm{~nm}$. The path-integrated number density is $1.8 \times 10^{13} \mathrm{~m}^{-2}$ at ion beam current of $45 \mathrm{~mA}$ and argon ion energy of $1,000 \mathrm{eV}$. The number density is proportional to the ion beam current, as expected. These results show the validity of the boron sensor for detecting sputtered boron atoms. Next, in order to achieve the required detection sensitivity and time response, we implement CRDS with a continuous-wave (cw) laser for enhanced sensitivity. The target was changed to manganese $(\lambda=403.07 \mathrm{~nm})$ instead of boron. The results show that a detection limit of per-pass absorbance of $0.6 \mathrm{ppm}$ for a 1-s measurement time. Scaling the experimental results and accounting for changes in laser system and mirror reflectivity indicate that the BN detection system should have sufficient signal-to noise for expected Hall thruster conditions.
\end{abstract}

Key Words: Hall thruster, lifetime, Cavity Ringdown Spectroscopy, erosion

\section{Nomenclature}

$\begin{array}{ll}A k i & : \text { Einstein } A \text { coefficient, } 1 / \mathrm{s} \\ A b s & : \text { Absorbance } \\ c & : \text { Speed of light, } 2.998 \times 10^{8} \mathrm{~m} / \mathrm{s} \\ E_{i} & : \text { Energy of state } i, \mathrm{~J} \\ E_{k} & : \text { Energy of state } k, \mathrm{~J} \\ g_{i} & : \text { Degeneracy of state } i \\ g_{k} & : \text { Degeneracy of state } k \\ k(v) & : \text { Absorption coefficient, } \mathrm{m}^{-1} \\ l & : \text { Length of the ring-down cavity, m } \\ N_{i} & : \text { Lower state concentration, } \mathrm{m}^{-3} \\ R & : \text { Mirror reflectivity } \\ S(t, \mathrm{v}) & : \text { Ring-down signal } \\ x & : \text { Position along the optical axis } \\ v & : \text { Laser frequency, Hz } \\ \tau & : \text { Ring-down time, s } \\ \tau & : \text { Empty cavity ring-down time, } \mathrm{s}\end{array}$

\section{Introduction}

Hall thrusters are a class of electric propulsion devices developed in the 1960 s to alleviate the thrust density limitation of ion thrusters. Because grids are not required to accelerate the ions, the thrusters are also attractive since they do not suffer from the large grid erosion rates of ion thrusters. Since 1971 two SPT-60 Hall thrusters have been used on the Meteor satellite and over 238 Hall thrusters have been operated in space on 48 spacecraft. Commercial Hall thrusters are now available in the US from Aerojet, Busek, and International Space Technologies Incorporated (ISTI). Space Systems/Loral flew Fakel SPT-100s on its MBSAT in 2004 while the ESA recently set endurance records for in-space Hall thruster operation with the PPS-1350-G used on the SMART-1 lunar mission. Although the specific impulse of Hall thrusters is considerably higher than that of chemical rockets, the thrust values are much lower. The tradeoff is due to the fact that the Hall thrusters (and other EP systems) derive their electrical power from the spacecraft. This means that Hall thrusters will have to operate for considerably longer durations than their chemical propulsion counterparts so that long engine life is critical. For example, a Hall thruster used for NSSK of a commercial spacecraft will have to operate for over 5,000 hours over the course of its mission ${ }^{1,2)}$.

The life-limiter for Hall thrusters is acceleration channel wall erosion ${ }^{3)}$. Not only does acceleration channel wall erosion lead ultimately to the end of thruster life once the wall is completely eroded through, but sputtered wall material can redeposit and form coatings on spacecraft surfaces such as solar arrays and sensor optics thereby posing a serious risk to spacecraft operation. Moreover, the effect of varying thruster operating parameters such as voltage and mass flow rate on lifetime is not well understood for Hall thruster operation. This lack of understanding makes developing a high-performance Hall thruster that is designed to span a wide range of operating conditions (e.g., voltage and mass flow rate) extremely challenging and expensive. Currently, the only way of verifying that a thruster has sufficient life for its mission 
is to operate it beyond its expected total thrust duration in a vacuum chamber in a life test. However, such life tests can cost several hundred thousand to millions of dollars and can tie up valuable vacuum facilities and engineers for several months. What is needed, therefore, is a method of measuring thruster erosion rates non-intrusively in real- or near-real-time. The erosion rate can be measured by probing the eroded wall material in the plume. Such measurements would allow simultaneous evaluation of the impact of Hall thruster design changes on performance and lifetime.

The ideal diagnostic for in situ thruster studies should have high sensitivity to measure low erosion rates, the possibility of integration to a thruster test-facility, and fast time-response to explore a range of operating conditions. Techniques such as weight loss ${ }^{4)}$, collector plates ${ }^{5)}$, quartz crystal microbalance ${ }^{6,7)}$, radioactive tracers ${ }^{8)}$, mass spectrometr $^{9)}$, and Rutherford backscattering ${ }^{10)}$, each have certain advantages and can be appropriate for material sputter characterization studies but none readily meets all of the above criteria. The need for a sensitive nonintrusive measurement suggests the use of optical techniques. Optical emission spectroscopy (OES) ${ }^{11,12)}$, laser induced fluorescence (LIF) ${ }^{13)}$ and multi-photon ionization coupled to a time of flight mass spectrometer ${ }^{14,15)}$ have been used for species-specific sputtering measurements. The use of LIF has been particularly extensive and has proven to be very effective for velocity measurement though challenging for quantitative number density. OES is attractive owing to its experimental simplicity but the analysis can be challenging since collisional-radiative modeling (or similar) is required to extract number densities ${ }^{16)}$. Owing to these limitations, Laser Absorption Spectroscopy (LAS) has been proposed for erosion rate measurement ${ }^{3}$. LAS is a non-intrusive optical method with the potential advantage of providing directly quantitative number density measurements (meaning it does not require external calibration). Furthermore, LAS is amenable to in situ studies which can be conducted in near real-time. Our previous research showed the possibility of nonintrusive near real-time erosion measurement by LAS in an anode layer type Hall thruster ${ }^{3}$; however, the approach didn't yield sufficient sensitivity for number density measurements at needed conditions. The approach presented here builds upon our previous development of cavity ring-down spectroscopy (CRDS) for sputtering measurements ${ }^{17-21)}$. Past research has developed the use of cavity ring-down spectroscopy (CRDS) to study sputtering of molybdenum, titanium and other species. CRDS is a path-enhanced laser absorption method that provides the ultra-high sensitivity required to measure low erosion rates.

Our current focus is to develop a CRDS boron sensor for Hall thruster erosion studies. Boron nitride $(\mathrm{BN})$ is generally used for the acceleration channels of magnetic layer type Hall thrusters. The measurement strategy is based upon probing boron atoms absorption line from their ground state which is in the vicinity of $250 \mathrm{~nm}$ in the ultraviolet. CRDS measurements of boron present challenges owing to reduced mirror reflectivities in the ultraviolet (mirrors with $\mathrm{R} \approx 0.996$ have been used in our work to date).

In this contribution, we present initial development of CRDS to study boron nitride. As a first step toward the boron sensor, we have performed proof of principle measurements of boron from BN targets using pulsedCRDS in a diagnostic chamber which allows elevated boron number densities. Next, in order to achieve the required detection sensitivity and time response, we implement CRDS with continuous-wave (cw) lasers for enhanced sensitivity. The target changed to manganese $(\lambda=403.07 \mathrm{~nm})$ instead of boron.

\section{Cavity Ring-Down Spectroscopy (CRDS)}

CRDS is a path-enhanced laser absorption method that provides the ultra-high sensitivity (down to sub-ppm levels per pass) ${ }^{22)}$. The technique is used extensively for trace-species measurement in flames, plasmas, and the atmosphere and we have developed its use for the study of sputtered particles in electric propulsion applications. The technique is directly quantifiable and can measure the ground states. Measuring ground states can be advantageous since these levels typically contain a large fraction of the overall species population and their population fractions are less affected by collisional and radiative rates.

As shown in Fig. 1, the basic idea is to introduce an absorbing species (i.e. the sputtered atoms) into a high finesse optical cavity formed from high-reflectivity (HR) mirrors. The probe laser beam is coupled into the optical cavity where it "bounces" many times back-and-forth between the mirrors. Owing to the high reflectivity, the light within the cavity makes many passes within the cavity (e.g. $\sim 10^{4}$ passes for $\mathrm{R} \sim 0.9999$ ), and the effective path length and thus sensitivity is greatly increased. A detector placed behind the cavity measures the temporal decay of optical intensity within the cavity. The difference in the temporal decay rate with and without the absorber (or with the laser tuned on/off the resonance) yields the sample concentration. The technique affords high sensitivity owing to a combination of long effective path length and insensitivity to laser energy fluctuations (since a rate is measured). Under appropriate conditions, the ring-down signal $S(t, \mathrm{n} v)$ decays single exponentially versus time as ${ }^{22,23)}$,

$$
\begin{aligned}
& S(t, v)=S_{0} \exp \left(-\frac{t}{\tau(v)}\right) \\
& \frac{1}{\tau(v)}=\frac{l}{c} \quad\left[\int k(x, v) d x+(1-R)\right]
\end{aligned}
$$

If the absorber is uniformly present over a column length $l_{a b s}$, then $\int k(x, \mathrm{n}) d x$ can be replaced with the product $k(\mathrm{n}) l_{a b s}$. In practice, the measured ring-down signal is

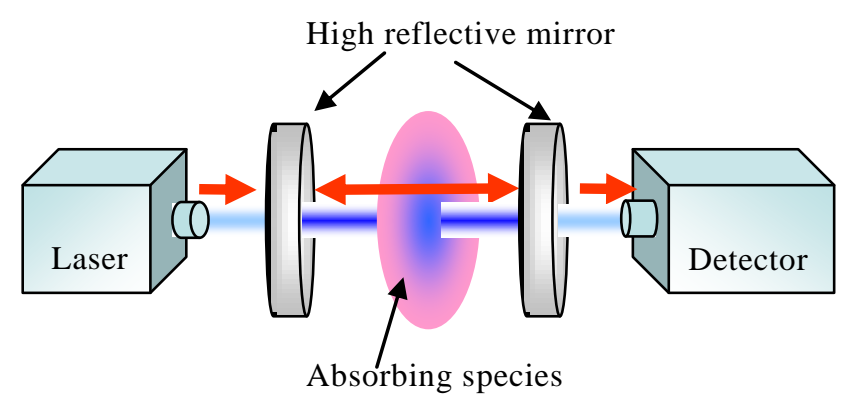

Fig. 1. The Basic idea of CRDS 
fitted with an exponential, and the ring-down time $\tau$ is extracted. Combining $\tau$ with the "empty cavity ring-down time", $\tau_{0}$ (which in practice is measured by detuning the laser) allows determination of the sample absorbance, $A b s(v)$, and absorption coefficient:

$$
\operatorname{Abs}(v)=\int k(x, v) d x=\frac{l}{c}\left[\frac{1}{\tau}-\frac{1}{\tau_{0}}\right]
$$

A commonly used approach is to scan the laser frequency across the absorption line and to measure the wavelength(or frequency-) integrated spectrum (i.e. the line area). Assuming the spectroscopic line parameters are known, the measured area $\int A b s(v) d v$ of a transition from lower state $i$ to upper state $k$ can be readily converted to the path-integrated concentration of the lower state $\int N_{i} d x$ as:

$$
\int N_{i} \mathrm{~d} x=\frac{v_{k i}^{2}}{c^{2}} 8 \pi \frac{g_{i}}{g_{k}} \frac{1}{A_{k i}}\left(\int A b s(v) d v\right)
$$

For cases where the spatial distribution of particles is non-uniform, actual concentration profiles can be determined from the path-integrated concentration in several ways. For rough approximation one can assume a uniform concentration profile over a known column length, $l_{a b s}$. Alternatively, Abel inversion or other inversion approaches based on inversion and modeled spatial profiles can be used ${ }^{24)}$. CRDS can also be used to extract velocity information from the measured spectral lineshapes ${ }^{21)}$.

\section{Boron measurement by Pulsed CRDS.}

We have performed proof of principle measurements of boron from $\mathrm{BN}$ target using pulsed CRDS in a diagnostic chamber allowing elevated boron number densities.

\subsection{Experimental Setup}

Figure 2 shows a schematic diagram of the bench-top sputtering apparatus employed for CRDS diagnostic development ${ }^{17)}$. The apparatus allows us to use CRDS to probe sputtered particles created by an ion beam incident upon a target. The key components are an ion beam and target, housed within a vacuum facility. A roughing and turbo-pump are used to bring the pressure to approximately $10^{-4} \mathrm{~Pa}$ under no-flow conditions. A small argon flow $(0.04 \mathrm{mg} / \mathrm{s})$ is used to feed the system. The ion beam is extracted from an $8-\mathrm{cm}$ diameter structurally integrated thruster obtained from NASA, and modified to operate on an inert gas, and to use thoriated tungsten filaments for both the main and neutralizer cathodes in place of the hollow cathodes used in the original design. The ion beam is masked to an active area of $8 \mathrm{~cm} \times 2.5 \mathrm{~cm}$ (with the $8 \mathrm{~cm}$ extent oriented parallel to the optical axis). Ion beam conditions were a beam current of $45 \mathrm{~mA}$ and beam voltage of $1200 \mathrm{~V}$ and the optical axis was roughly 1 $\mathrm{cm}$ above the target. The CRDS set-up, schematically shown in Fig.2, uses a broadly tunable optical parametric oscillator (OPO) laser. For the boron experiments we use the doubled-signal beam as the light source in the $250 \mathrm{~nm}$ region. Laser parameters are: repetition rate $=10 \mathrm{~Hz}$, pulse width $\sim 7 \mathrm{~ns}$, pulse energy $\sim 2 \mathrm{~mJ}$, and linewidth

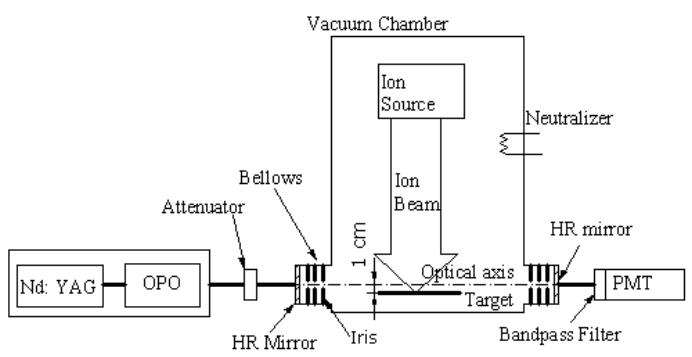

Fig. 2. Schematic diagram of sputtering apparatus and CRDS system

\begin{tabular}{ccc} 
Table 1. & Transition data for boron (from NIST database ${ }^{25)}$ ). \\
\hline$\lambda, \mathrm{nm}$ (Air) & 249.677 & 249.773 \\
\hline$E_{i}, \mathrm{eV}$ & 0 & 0.001893 \\
$E_{k}, \mathrm{eV}$ & 4.9642836 & 4.9642836 \\
$A_{k i,} \mathrm{~s}^{-1}$ & $1.2 \times 10^{8}$ & $2.4 \times 10^{8}$ \\
\hline
\end{tabular}

$\sim 0.002 \mathrm{~nm}$. We use a linear ring-down cavity of $0.75 \mathrm{~m}$ length with $1 \mathrm{~m}$ radius-of-curvature mirrors. In order to prevent possible saturation effects, the laser energy is reduced with an attenuator to $\sim 100 \mu \mathrm{J} /$ pulse prior to cavity injection. The ring-down signal is collected behind the output mirror with a fast photomultiplier tube (PMT). Ring-down signals are fit between $90 \%-10 \%$ of the peak amplitude. We use area (frequency-integrated) measurements of absorbance in our analyses.

Table 1 shows energy levels, spontaneous emission rates (Einstein A coefficients), and wavelengths for the targeted line. The ground term for this species has two distinct levels: $2 s^{2} P_{1 / 2}{ }^{0}$ (at $0.00000 \mathrm{eV}$ ) and $2 s^{2} P_{3 / 2}{ }^{0}$ (at $0.00189 \mathrm{eV}$ ). As a result, fine-structure splitting results in two distinct B I absorption lines near $250 \mathrm{~nm}$ : the $2 s^{2} P_{1 / 2}{ }^{0}$ $\rightarrow 3 s^{2} S_{1 / 2}$ absorption at $249.677 \mathrm{~nm}$ (Air), and the $2 s^{2} P_{3 / 2}$

${ }^{0} \rightarrow 3 s^{2} S_{1 / 2}$ absorption at $249.773 \mathrm{~nm}$ (Air). The targeted lines are selected based on their optical accessibility and high absorption strength. The next lowest energy level is above $3 \mathrm{eV}$ so that to a good approximation all population will reside in the split ground state, meaning that the measured states will provide a direct measure of the overall boron population.

\subsection{Results}

Figure 3 shows the corresponding absorbance spectrum after baseline fitting and subtraction along with a best-fit Voigt peak for the $249.773 \mathrm{~nm}$ line. The BN sample is HBC grade from General Electric's Advanced Ceramics. The BN sample used was $10 \mathrm{~cm}$ by $10 \mathrm{~cm}$ square with thickness of $2 \mathrm{~mm}$. Initial studies of dependence of boron path-integrated number density versus beam current at constant beam voltage of $1000 \mathrm{~V}$ have been performed and are shown in Fig. 5. The path-integrated number densities are determined from the absorption lines areas as found from Voigt fits to the measured spectra. Assuming a column length of $10 \mathrm{~cm}$ (corresponding to the target dimension), the rough estimated number density of boron is $1 \times 10^{13} \mathrm{~m}^{-3}$ at ion beam of $45 \mathrm{~mA}$ and beam voltage of 


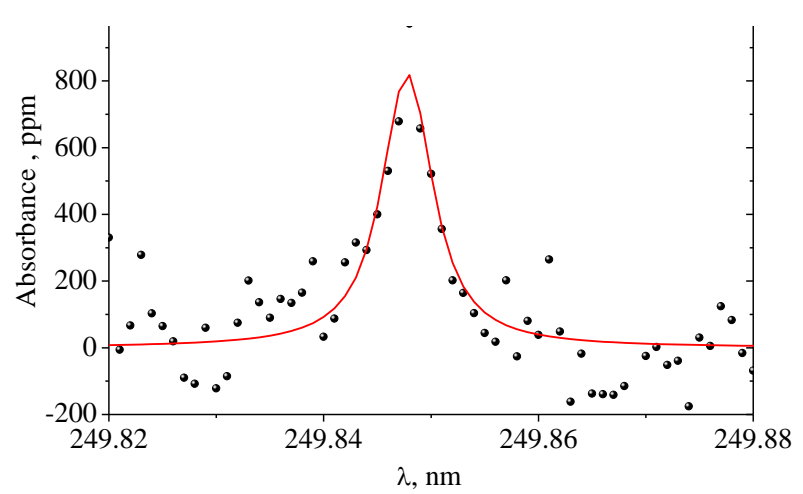

Fig. 3 CRDS measurement of boron from BN target.

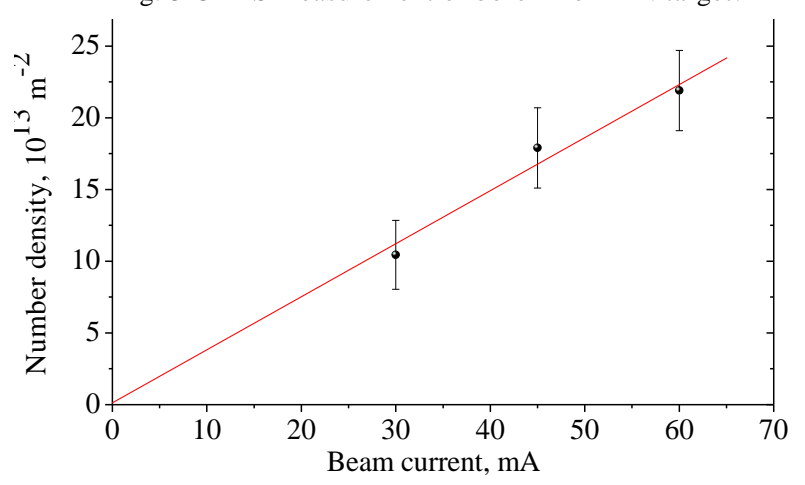

Fig. 4. Boron number density versus ion beam current.

$1,200 \mathrm{~V}$. The concentration is expected to be proportional to the beam current, as is observed in Fig. 4.

These results show the validity of the CRDS boron sensor using ultraviolet probe laser and the availability of this sensor for Hall thruster erosion studies, though the $\mathrm{SN}$ ratio must be improved.

\section{Real-time monitoring system by cw-CRDS}

We have performed proof of sensitivity and time response of the sputtered material sensor using cw-CRDS in a diagnostic chamber. The target changed to manganese from boron, since the cw- CRDS boron sensor has a lot of challenges. The future BN system will use different laser and mirrors, but that this experiment serves to demonstrate cw-CRDS for sputter measurements, including examination of practical issues such as cavity alignment and mirror contamination.

\subsection{Experimental Setup}

We use a tunable diode laser with external cavity (ECLD) to measure the transition line of manganese at $403.076 \mathrm{~nm}$ (Air). The transition data for this measurement are shown in Table 2, as quoted from the NIST database ${ }^{25)}$. The modehop free tuning range of the laser is about $30 \mathrm{GHz}$ and the laser linewidth is less than 5 MHz. An optical isolator and Single Mode FC/APC fiber are used to prevent back reflections into the laser. An aspheric lens is used at the fiber exit to collimate the beam and to match the beam to $\mathrm{TEM}_{00}$ mode of the cavity.
A threshold detection circuit and an acousto-optic modulator (AOM) are used to extinguish the incoming laser beam. The combined time response of the threshold circuit and AOM firing is less than $400 \mathrm{~ns}$. A photomultiplier tube is used as a detector and positioned behind the cavity. A dielectric interference filter and an iris are used to suppress background light and the emission from the plasma. Ring-down signals are measured with a $20 \mathrm{MHz}$ 12-bit analog-to-digital acquisition board connected to a personal computer, and a custom Labview program is used for exponential fitting with the nonlinear Levenberg-Marquardt fit. A solid etalon (free spectral range $=2.26 \mathrm{GHz}$ ) is used as a frequency reference.

The optical cavity length is $0.83 \mathrm{~m}$ and is formed by a pair of high reflectivity mirrors (Los Gatos Research, $\mathrm{R}>99.995$ ), each $25.4 \mathrm{~mm}$ in diameter with radius-of-curvature of $1 \mathrm{~m}$. We typically operate with empty-cavity ring-down times of $\sim 20 \mu$ s, corresponding to R 99.986\% (close to manufacturer's specifications). The mirrors are held in custom mounts affixed to the end of extender arms connected to the vacuum chamber. The mounts are mechanically and thermally isolated from the main chamber body by using bellows in line with the arms. A series of irises (diameter $\sim 5 \mathrm{~mm}$ ) are set to the arms in order to prevent the mirrors from deposition of sputtered particles. With this method, minimal degradation of mirror reflectivity has been observed ( 5-10 ppm/hour). Improved shielding and/or periodic cleaning would be required for longer term operation.

For demonstrating the real-time monitoring of the sputtered material, a multilayer target comprised of alternating layers of $\mathrm{Mn} / \mathrm{Fe}$ (500 $\AA$ thick) and titanium $(200 \AA)$ are used. The layers were sputter deposited onto a silicon wafer substrate. There is a top(outer) layer of titanium (3000 $\AA$ ) which must be sputtered before reaching the alternating layers containing $\mathrm{Mn}$. The dimensions of the target surface were $5 \mathrm{~mm} \times 5 \mathrm{~mm}$.

Table 2. Transition data for manganese (from NIST database).

\begin{tabular}{cc}
\hline$\lambda, \mathrm{nm}(\mathrm{Air})$ & 403.076 \\
\hline$E_{i}, \mathrm{eV}$ & 0 \\
$E_{k}, \mathrm{eV}$ & 3.075 \\
$A_{k i,} \mathrm{~s}^{-1}$ & $1.7 \times 10^{7}$ \\
$g_{i}$ & 6 \\
$g_{k}$ & 8 \\
\hline
\end{tabular}

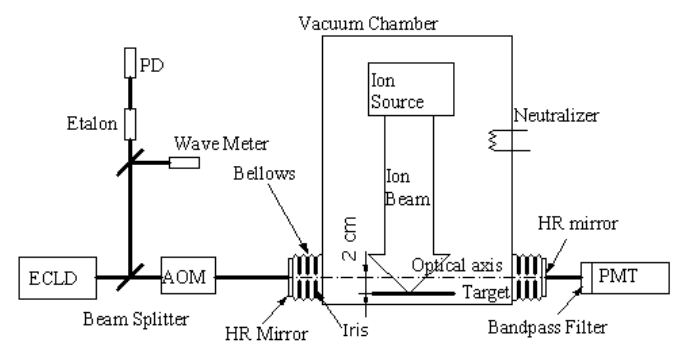

Fig. 5. Schematic diagram of real-time monitoring system 


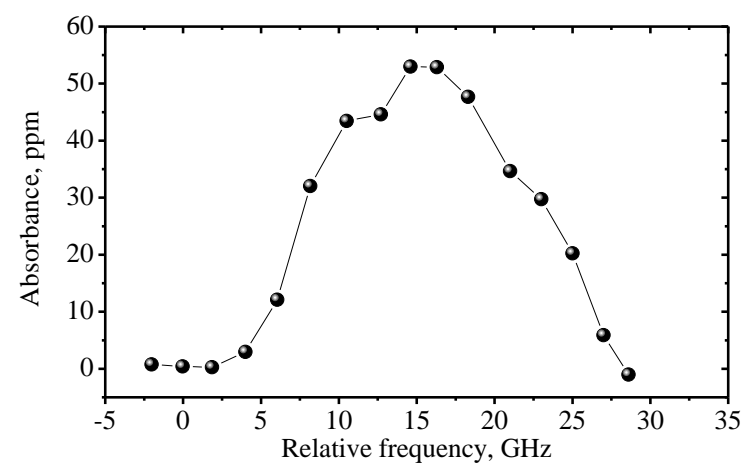

Fig. 6. Absorption profile of target spectrum.

Concentration measurements are determined from the area of absorption spectra, as shown in Fig. 6. We have subtracted a small baseline contribution $\left(\sim 10^{8} \mathrm{~cm}^{-2}\right)$ associated with residual $\mathrm{Mn}$ present in the vacuum chamber. In this case, the diode laser scan interval is 35 $\mathrm{GHz}$ and scan duration is $0.5 \mathrm{~s}$. The ion beam current and voltage were $120 \mathrm{~mA}$ and $750 \mathrm{~V}$ respectively. The optical axis is $2 \mathrm{~cm}$ above the target. To construct the spectrum we use a binning approach where the frequency axis is divided into a series of bins, each with width 2 $\mathrm{GHz}$, and signals falling within the bin are averaged. The slight thermal drift of the ring-down cavity over time actually aids in distributing the measurements more uniformly within the bins. The wavelength-integrated area of the spectrum yields the path-integrated concentration.

\subsection{Result}

Figure 7 shows the time dependence of manganese concentration deduced from the multilayer target. The cw-CRDS detection can readily detect the appearance and ending of the Mn-containing layers. The rounded profile of each layer is primarily owing to the non-uniformity of the ion beam current density distribution, which causes non-uniform sputter rates over the target area so that the layers start and finish at slightly different times at different locations on the target. For this reason, earlier tests with larger targets (e.g. $4 \mathrm{~cm} \mathrm{x} 2 \mathrm{~cm}$ ) failed to detect any clear $\mathrm{Mn} / \mathrm{Ti}$ boundary. The time duration of each $\mathrm{Mn} / \mathrm{Fe}$ layer is approximately 40 seconds, corresponding to etching rates of approximately $\sim 13 \AA$ /s for the $500 \AA$ thick layers The observed etch rates of $\sim 10 \AA / s$ agrees reasonably with a simple numerical estimate of $\sim 23 \AA / \mathrm{s}$ based on the average ion current density $\left(j=2.0 \mathrm{~mA} / \mathrm{cm}^{2}\right)$, expected sputter yield $(\mathrm{Y}=1.51$ atoms/ion based on averaging single-species yields of $\mathrm{Y}_{\mathrm{Mn}}=2.01$ atoms/ion and $\mathrm{Y}_{\mathrm{Fe}}=1.00$ atoms/ion for $600 \mathrm{eV}$ argon ions ${ }^{12}$ ), effective molar mass and density (using average of $\mathrm{Mn}$ and $\mathrm{Fe}$ values), and the target thickness. The experimental titanium etch rate of $\sim 5 \AA / s(\sim 200 \AA / 40$ s $)$ is also reasonably consistent with a similar numerical estimate of etch rate of $\sim 7 \AA / \mathrm{s}$ (found analogous to that described above but with titanium material properties and an expected sputter yield of $\mathrm{Y}_{\mathrm{Ti}}=0.53$ atoms/ion for $600 \mathrm{eV}$ $\operatorname{argon}$ ions $^{12}$ ).

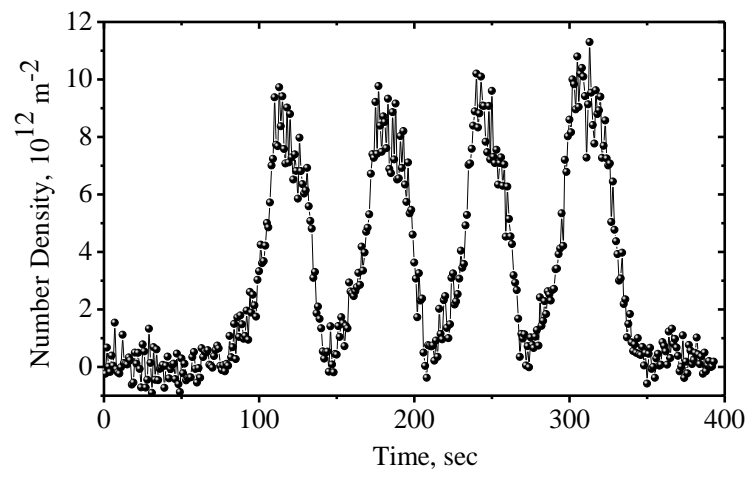

Fig. 7. Time dependence of manganese concentration.

The cw-CRDS real-time sputter monitoring system reported here is, to the best of our knowledge, the first such demonstration. As discussed below, the sensitivity of the system is very adequate for expected Hall thruster erosion conditions. In terms of optical sensitivity, the minimum detectable absorbance of our system is $2 \mathrm{ppm}$ per laser shot, and the measurement repetition rate is 200 Hz. Therefore, the sensitivity can be estimated as $1.4 \times 10^{-8} \mathrm{~cm}^{-1} \mathrm{~Hz}^{-1 / 2}$ (assuming a sample length of $10 \mathrm{~cm}$ ). Equivalently, the detection limit of per-pass absorbance at a fixed wavelength is $0.14 \mathrm{ppm}$ for a $1-\mathrm{s}$ measurement time. However, when performing manganese measurements, the actual 1-s detection limit was $0.6 \mathrm{ppm}$ with the degradation in sensitivity being due to the method used for scanning and binning the laser wavelength. Using this latter value, and considering the reduced mirror reflectivity at $250 \mathrm{~nm}(\mathrm{R} \sim 0.996$ at $250 \mathrm{~nm}$ versus $\mathrm{R} \sim 0.99986 \%$ at $403 \mathrm{~nm}$ ), the corresponding detection limit of $250 \mathrm{~nm}$ boron CRDS system would be $\sim 20$ ppm for a 1-s measurement time.

Based on scaling of wall erosion rates modeled by Yim et al. ${ }^{26)}$, we predict a path integrated number density at the thruster exit plane of boron of $10^{13}-10^{14} \mathrm{~m}^{-2}$. The corresponding peak absorbance signal is $\sim 40-400 \mathrm{ppm}$ if we assume the same linewidth of $\sim 0.005 \mathrm{~nm}$ as found in the pulsed CRDS measurements (shown in Fig.3). Thus, we anticipate that our CRDS detection system will allow sputter measurements with a signal-to-noise ratio of 2 to 20 for one-second measurement. We will further improved the signal to noise ratio by increasing the measurement repetition rate. Using a PZT on the cavity mirror to bringing the cavity into resonance with the $\operatorname{laser}^{27)}$ will improve the repetition rate up to $5000 \mathrm{~Hz}$, meaning the detection limit of the boron sensor will be $4 \mathrm{ppm}$ for one second. Correspondingly, the signal-to-noise ratio will improve to 10 to 100 (depending on boron concentration). This sensitivity of the BN system should be sufficiently high for real-time monitoring of Hall thruster erosion.

\section{Summary}

For the building a real-time or near real-time erosion monitoring system in Hall thrusters, we performed two experiments. 
First, Number density measurements of boron atoms sputtered from BN targets by argon ions were performed. The measurements employ ultraviolet laser based pulsed cavity ring-down spectroscopy (CRDS) in the region of ultraviolet, $250 \mathrm{~nm}$. Measurements in this spectral region are challenging one since the reflectivity of available mirror at wavelength of $250 \mathrm{~nm}$ is $\mathrm{R} 0.996$, this is relatively low comparison with that of a mirror adopted conventional CRDS measurements. The measured number density at ion energy of $1,000 \mathrm{~V}$ and ion beam current of $45 \mathrm{~mA}$ was $1 \times 10^{13} \mathrm{~m}-3$, which is in reasonable agreement with expectations from modeling and past research. Dependence of sputtered particle number density on ion beam current was also measured and found to be in accord with expectations. The boron measurements provide proof of principle for a $\mathrm{BN}$ erosion sensor that we will develop.

Next, time variation of the number density of manganese atoms sputtered from multilayer targets by argon ions were measured in order to build real-time monitoring system of sputtered material. For the demand on time response and sensitivity, cw-CRDS is adopted. The observed etch rates of $\sim 10 \AA /$ s agrees reasonably with a simple numerical estimate of $\sim 23 \AA /$ s. it means the time response of this sensor is adequate. A detection limit of per-pass absorbance is $0.6 \mathrm{ppm}$ for a 1 -s measurement time. Considering the reduced mirror reflectivity at $\lambda=250$ $\mathrm{nm}$, the detection limit is estimated as $20 \mathrm{ppm}$ for 1 second measurement. The estimated signal level is 40-400 $\mathrm{ppm}$, therefore, this signal to noise ratio is estimated as 2-20 for 1 second measurement. It could be improved to $10-100$ by using a PZT.

Signal levels, time response and signal-to-noise of the cw-CRDS sensor have been examined and should allow near real time measurements of sputter erosion in Hall thrusters, thereby providing a new and powerful tool for sputter erosion and lifetime studies.

\section{Acknowledgements}

The present work was supported by a Grant-in-Aid for Scientific Research (S), No. 16106012, sponsored by the Japan Society for the Promotion of Science, Japan.

\section{References}

1) Garner, C. E., Polk, J. E., Goodfellow, K. D., and Brophy, J. R.: Performance Evaluation and Life Testing of the SPT-100, IEPC paper 93-091, Sept. 1993.

2) Garner, C. E., Brophy, J. R., Polk, J. E., and Pless, L. C.: A $5730-\mathrm{Hr}$ Cyclic Endurance Test of the SPT-100, AIAA paper 95-2667, July 1995.

3) Yamamoto, N., Yokota, S., Matsui, M., Komurasaki, K., Arakawa, Y.: Measurement of Erosion Rate by Absorption Spectroscopy in a Hall Thruster, Review of Scientific Instruments, 76, Issue 8, 083111,(2005)

4) Yalin, A. P., Surla, V., Farnell, C., Butweiller, M., and Williams, J. D.: Sputtering studies of multi-component materials by weight loss and cavity ring-down spectroscopy, AIAA paper 2006-4338, July, 2006.

5) Tsuge, H. and Esho, S.: Angular distribution of sputtered atoms from polycrystalline metal targets, J. Appl. Phys. 52 (1981) pp.4391-4395.
6) Yalin, A. P., Williams, J. D., Surla, V. and Zoerb, K. A. Differential Sputter Yield Profiles of Molybdenum due to Bombardment by Low Energy Xenon Ions at Normal and Oblique Incidence, J. Phys. D: Appl. Phys. 40 (2007) pp. 3194-3202.

7) Mannami, M., Kimura, K., and Kyoshima, A.: Angular distribution measurements of sputtered $\mathrm{Au}$ atoms with quartz oscillator microbalances, Nuclear Instruments and Methods, 185 (1981) pp.533-537

8) Kundu, S., Ghose, D., Basu, D., and Karmohapatro, S. B.: The angular distribution of sputtered silver atoms, Nuclear Instruments and Methods in Physics Research B 12 (1985) pp.352-357.

9) Wucher, A. and Reuter, W.: Angular distribution of sputtered particles from metals and alloys, J. Vac. Sci. Tech. A 6, 4 (1988) pp.2316-2318.

10) Mantenieks, M., Foster, J., Ray P., Shutthanandan, S., and Thevuthasan, T.: Low energy xenon ion sputtering yield measurements, IEPC paper 01-309, 2001

11) Andersen, N., B. Andresen, and Veje, E.: Atomic excitations in sputtering processes, Radiation Effects, 60 (1982) pp.119-127.

12) Doerner, R.P., Whyte, D.G., and Goebel, D.M.: Sputtering yield measurements during low energy xenonplasma bombardment, J. App. Phys. 93(9) (2003) pp.5816-5823.

13) Pellin, M.J., Wright, R.B., and Gruen, D.M.: Laser fluorescence spectroscopy of sputtered zirconium atoms, $J$. Chem. Phys. 74 (1981) pp.6448-6457

14) Nicolussi, G., et al.: Formation of metastable excited $\mathrm{Ti}$ and $\mathrm{Ni}$ atoms during sputtering, Phys. Rev. B 51, 14 1995, pp.8779-8788.

15) Goehlich, A.: Investigation of time-of-flight and energy distributions of atoms and molecules sputtered from oxygen-covered metal surfaces by laser techniques, Appl. Phys. A. 72 (2001) pp.523-529.

16) Karabadzhak, G.F., Semenkin, A.V., Solodukhin, A.E. and O.S.Tverdokhlebov,: Evaluation of Impurity Composition and Content in the TAL at Various Operating Regimes, IEPC paper-2005-147,2005.

17) Surla,V. Wilbur, P.J. Williams, J.D. Johnson,M. Yalin, A.P.: Sputter Erosion Measurements of Titanium and Molybdenum by Cavity Ring-Down Spectroscopy, Review of Scientific Instruments, 75, 9, (2004) pp. 3025-3030.

18) Yalin, A.P., Surla, V., Butweiller, M., Williams, J.D.: Detection of Sputtered Metals using Cavity Ring-Down Spectroscopy, Applied Optics, 44, 30 (2005) pp. 6496-6505.

19) Yalin, A.P., Surla, V.: Determination of Number Density and Velocity of Sputtered Particles by Cavity Ring-Down Spectroscopy, IEPC paper 2005-300, Oct. 2005.

20) Yalin, A.P., Surla, V.: Velocity Measurements by Cavity Ring-Down Spectroscopy, Optics Letters, 30, (2005) pp. 3219-3221

21) Busch K. W. and Busch M.A. :Cavity-Ringdown Spectroscopy, American Chemical Society, Washington DC,(1999) pp.7-19.

22) Zalicki, P. and Zare, R.N.: Cavity ring-down spectroscopy for quantitative absorption measurements, J. of Chemical Physics, 102, 7 (1995) pp. 2708-2717.

23) Yalin, A.P., and Zare, R.N.: Effect of Laser Lineshape on the Quantitative Analysis of Cavity Ring-Down Signals, Laser Physics, 12, 8, (2002) pp. 1065-1072.

24) Holloway, J., Shannon, S., Sepke, S., and Brake, M.: A reconstruction algorithm for a spatially resolved plasma optical emission spectroscopy sensor, J. of Quantitative Spectroscopy and Radiative Transfer, 68, 1 (2001), pp.101-115

25) http://www.physics.nist.gov/cgi-bin/ASD/lines1.pl

26) Yim, J.T., Keidar, M., and Boyd. I.D.: An investigation of factors involved in Hall thruster wall erosion modeling, AIAA paper 2006-4657,(2006) July, 2006

27) Debecker, I., Mohamed, A. K., Romanini, D.:High-speed cavity ringdown spectroscopy with increased spectral resolution by simultaneous laser and cavity tuning, Optics Express, 13, (2005) pp. 2906-2915 